\title{
Binary star analysis with intrinsic pulsation
}

\author{
R.E. Wilson ${ }^{1}$, W. Van Hamme ${ }^{2}$ and G.J. Peters ${ }^{3}$ \\ 1 Astronomy Department, University of Florida, Gainesville, FL 32611, \\ U.S.A. (E-mail: rewilson@ufl.edu) \\ 2 Department of Physics, Florida International University, Miami, FL 33199, \\ U.S.A. (E-mail: vanhamme@fiu.edu) \\ 3 Department of Astronomy, University of Southern California, Los Angeles, \\ CA 90089, U.S.A. (E-mail: gpeters@usc.edu)
}

Received: October 8, 2019; Accepted: December 17, 2019

\begin{abstract}
Strategies are introduced for coherent measurement of pulsational waveforms, amplitudes, periods, and period change rates, where 'coherent' means that the model stars indeed pulsate, with no artificial alternation between pulsation without binary effects and binary effects without pulsation. Capabilities of the more advanced eclipsing binary (EB) light curve models are in place, such as simultaneous solutions of multiband data and radial velocities with inclusion of tides, mutual irradiation, and proper datapoint weighting. An application to the Algol-type EB V1352 Tau exhibits possibilities.
\end{abstract}

Key words: binaries: eclipsing, binaries: spectroscopic, stars: pulsating

\section{Introduction}

This contribution describes an analytic model to measure parameters from light and radial velocity (RV) curves of radially pulsating binary components. The modeled stars actually pulsate and there is no need for removal of pulsational variation from the data, in contrast to the pulsation subtraction schemes of most binary system pulsation models. Accordingly, all variation phenomena are handled within the model and the data are analyzed as observed, whether they are fluxes, magnitudes, or RVs. Tidal and rotational distortions of star figures and their accompanying surface brightness variations are built in, and there is no limitation to spherical stars. A major advantage and driver of the development has been that eclipse effects on pulsation arise as in the real stellar systems, since pulsations are eclipsed along with the model stars, of which they are an intrinsic feature, and geometric pulsations also affect eclipses via changes in eclipsing horizons. A present simplification is that pulsation is assumed slow enough to be computed as a sequence of equilibrium states. Although no pulsational dynamics are now in the model, they can be added later. Many capabilities of the more advanced EB light/velocity curve models are in place within the direct (curves from parameters) and inverse (parameters from curves) pulsational computer programs, which are extensions of the corresponding Wilson-Devinney (W-D) 
public modeling programs (Wilson and Devinney, 1971; Wilson and Sofia, 1976; Wilson, 1979, 1990, 2007, 2008; Wilson and Van Hamme, 2010; Wilson, 2012; Wilson and Van Hamme, 2014) such as proper datapoint weighting, simultaneous solution of RV and multi-band curves, tidal/rotational star figures, reflection effect, eclipses, etc.

Further intuitive comments on basic pulsational analysis for binaries do not fit within the space limit, but see Riazi and Abedi (2006) for coupled illustrations and explanations from their work on AB Cas and synthetic binaries. Kopal (1982a) and Kopal (1982b) cover historical items as well as advanced issues such as precession and nutation of non-aligned rotational axes. The impressive mathematical developments in these two papers remain to be applied in published binary system analyses.

\section{What else does the development accomplish?}

Several features add to the model's impersonal parameter adjustment performance from unaltered light/RV curve data - for systems with tides, rotational distortion, mutual irradiation, eccentric orbits, and other tight binary phenomena. Among these is coherent and impersonal resolution of a pulsation waveform into a geometric and a radiative part. Color information from multiple photometric bands can improve the separation of geometric and radiative behavior. Also, mathematics (Wilson, 2005) developed for orbital ephemerides $\left(T_{0}, P\right.$, $d P / d t)$, applies as well to pulsation ephemerides, and enhances both areas of ephemeris accuracy considerably, especially for $d P / d t$.

\section{How are the computations done?}

Coherent separation of geometric and radiative behavior is deduced from observations (Least Squares criterion) for each recognized periodicity, allowing comparisons with structural pulsation computations. Implementation is via multiplicative waveforms in mean radius (geometric) and mean effective temperature (radiative), each being a Fourier series in a fundamental and two harmonics. Of course, more harmonics can be added if needed. The Fourier coefficients are parameters to be determined from the data. The leading constant term is fixed at unity due to the series' multiplicative role. Thus the series evaluates to unity if all the determinable coefficients are zero (no pulsation). Multi-mode pulsation is handled by having additional periodicities in a given submission (thus more coefficients) or by successive applications, each with its initial parameter estimates. The geometric multiplier $\left(R_{\text {wave }}\right)$ is converted to a volume multiplier at given pulsation phase via $V=R_{\text {wave }}^{3}$. Next $V$ (phase) is converted to potential(phase), the star figure is computed as an equipotential [see Wilson (1979)], and local effective gravities are computed from the local potential gradients. Then, light or magnitude or RV are computed by appropriate integra- 
Table 1. V1352 Tau Solution Parameters

\begin{tabular}{lc}
\hline \hline$a\left(R_{\odot}\right)$ & 6.66924 \\
$i(\mathrm{deg})$ & $89.82 \pm 0.23$ \\
$T_{1}(\mathrm{~K})$ & 9000 \\
$T_{2}(\mathrm{~K})$ & $5819 \pm 23$ \\
$\Omega_{1}$ & $5.4689 \pm 0.0079$ \\
$\Omega_{2}$ & 2.25374 \\
$m_{2} / m_{1}$ & $0.20850 \pm 0.00054$ \\
$T_{0}(\mathrm{BJD})$ & $2457819.48312 \pm 0.00028$ \\
$P_{0}(\mathrm{~d})$ & $6.909673 \pm 0.000040$ \\
$L_{1} /\left(L_{1}+L_{2}\right)($ KEPLERband $)$ & $0.76620 \pm 0.00044$ \\
$\ell_{3}($ KEPLERband) & $0.00763 \pm 0.00057$ \\
$T_{0}$ puls $(\mathrm{BJD})$ & $2457860.1086 \pm 0098$ \\
$P_{\text {puls }}(\mathrm{d})$ & $0.0917305 \pm 0.0000021$ \\
$d P_{\text {puls }} / d t$ & $+0.37 \pm 1.16 \times 10^{-7}$ \\
Radius $\cos \theta \operatorname{coefficient}$ & $-0.0025 \pm 0.0014$ \\
Radius $\sin \theta \operatorname{coefficient}$ & $-0.0014 \pm 0.0017$ \\
Temperature cos $\theta$ coefficient & $-0.0007 \pm 0.0014$ \\
Temperature $\sin \theta$ coefficient & $-0.0017 \pm 0.0011$ \\
\hline \hline
\end{tabular}

tions over the surface. So the procedure does not just fit a wave to a light curve but operates at a deeper conceptual level. Note that all W-D model capabilities are in place, such as solution constraints, three kinds of weighting, solutions optionally in absolute units, direct distance estimation, etc.

\section{Initial application: V1352 Tauri}

The KEPLER mission light curve of V1352 Tau is a good start-up example for a pulsation model due to the binary's total-annular eclipses, high orbital inclination, and well resolved, steady pulsations. The observations cover 11 cycles of the 6.909673-day orbital period over 80 days, with a typical cycle shown in Fig. 1. The separate geometric and temperature parts of the overall solution within one of the maxima are compared with the observations in Fig. 2. Fig. 3 has the combined and separate waves on a magnified scale. $P_{\text {puls }}$ is measured to be $0.0917305 \pm 0.0000021$ days, although the pulsations are not seen in existing ground based datasets. $d P_{\text {puls }} / d t$ is nil within its uncertainty. A full list of parameter values is in Table 1 . A small amount of third light, $0.0076 \pm 0.0006$ of maximum system light, is indicated. Power spectra of light curve residuals show V1352 Tau to be a multi-mode pulsator with several periods longer than the orbital cycle. At least one likely periodic variation extends beyond KEPLER's timewise observing window. Much more pulsational analysis will surely follow these preliminary results. 


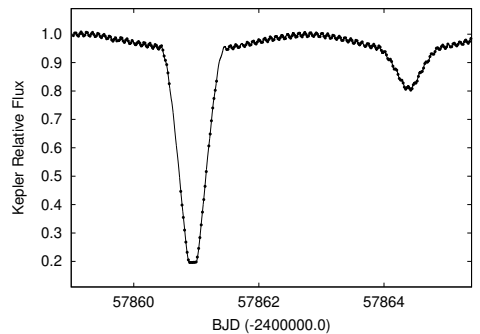

Figure 1. One orbit cycle of the V1352 Tau KEPLER light curve with solution curve.
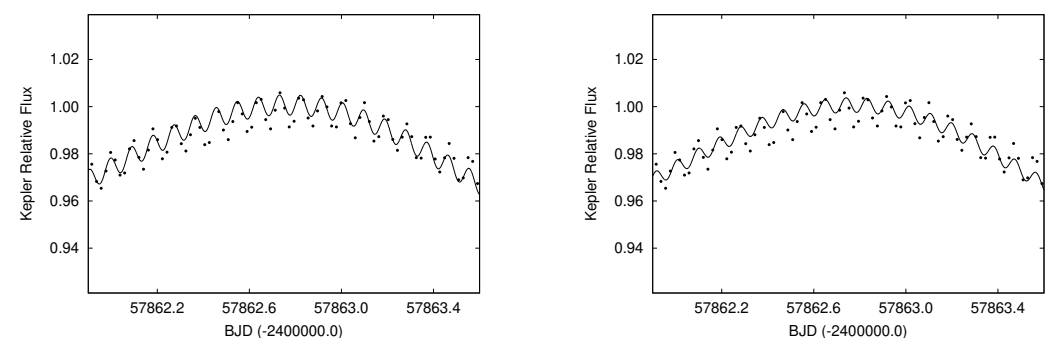

Figure 2. Pulsational lightcurve waveform in one of the maxima due only to geometric (i.e. mean radius) variation (left panel), and due only to temperature variation (right panel). Neither phenomenon alone accounts for the full variation.
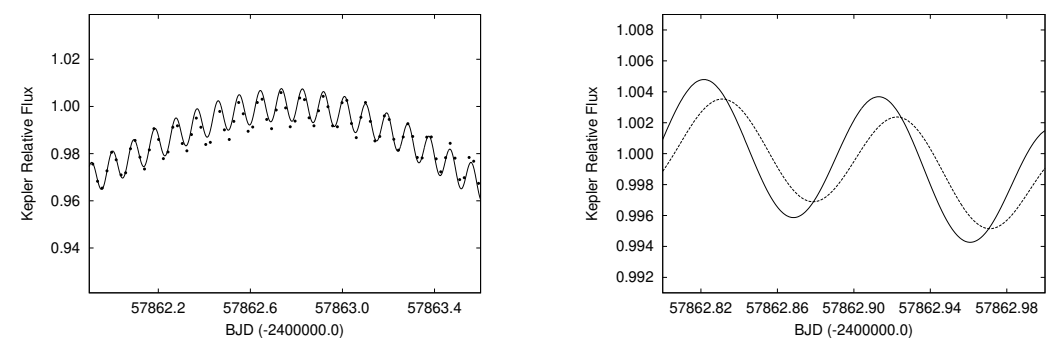

Figure 3. The lightcurve waveform in one of the maxima that results from the combined geometric and radiative pulsational variations (left panel). The right panel shows the separate geometric (continuous curve) and radiative (dashed curve) waveforms over about two pulsation cycles. 


\section{References}

Kopal, Z.: 1982a, Astrophys. Space Sci. 87, 149

Kopal, Z.: 1982b, Astrophys. Space Sci. 88, 313

Riazi, N., Abedi, A.: 2006, New Astr. 11, 514

Wilson, R.E.: 1979, Astrophys. J. 234, 1054

Wilson, R.E.: 1990, Astrophys. J. 356, 613

Wilson, R.E.: 2005, Astrophys. Space Sci. 296, 197

Wilson, R.E.: 2007, ASPC 362, 3

Wilson, R.E.: 2008, Astrophys. J. 672, 575

Wilson, R.E.: 2012, JASS 29, 115

Wilson, R.E., Devinney, E.J.: 1971, Astrophys. J. 166, 605

Wilson, R.E., Sofia, S.: 1976, Astrophys. J. 203, 182

Wilson, R.E., Van Hamme, W.: 2010, ASPC 435, 45

Wilson, R.E., Van Hamme, W.: 2014, Astrophys. J. 780, 151 